\title{
Geothermobarometer based on coexisting garnet-orthopyroxene-plagioclase-quartz equilibria
}

\author{
*Harel Thomas ${ }^{1}$ and Lalu P. Paudel ${ }^{2}$ \\ ${ }^{1}$ Department of Applied Geology, Dr. H. S. G. University, Sagar (MP), India \\ ${ }^{2}$ Central Department of Geology, Tribhuvan University, Kirtipur, Kathmandu, Nepal \\ (*E-mail: harelthomas@yahoo.com)
}

\begin{abstract}
The garnet-orthopyroxene-plagolcase-quartz pairs are commonly found in the assemblages of basic granulites/charnockite and hence are suitable for estimating equilibrium temperature and pressure of most metamorphic rocks. The temperature based on garnet-orthopyroxene Fe-Mg exchange reaction and pressure based on net transfer reaction of garnet-orthopyroxeneplagioclase-quartz equilibria, mainly based on reactions, using the equation given by different workers.

i. $1 / 2$ Ferrosilite $+1 / 3$ Pyrope $=1 / 2$ Enstatite $+1 / 3$ Almandine.

ii. Ferrosilite + Anorthite $=2 / 3$ Almandine $+1 / 3$ Grossularite + Quartz

iii. Enstatite + Anorthite $=2 / 3$ Pyrope $+1 / 3$ Grossularite + Quartz.

The author developed software in visual basic with the executable code MPET3.EXE
\end{abstract}

Keywords: Exchange reaction, Net transfer reaction, Geothermobarometer, Granulite

Received: 15 September 2015

Revision accepted: 5 May 2016

\section{INTRODUCTION}

One of the main goal of metamorphic petrology is to determine the pressure-temperature evolution of rocks in metamorphic terrains. Within this frame work, application of "geothermobarometry" in estimating the temperature and pressure conditions at which metamorphic rocks were once equilibrated has gained remarkable recognition in recent years. Impressive thermodynamic data sets and constantly improving activity models of minerals have substantially contributed to the success of rapidly evolving network (Knowledge) of (on) geothermobarometry. These P-T sensors have thus opened new vista for metamorphic petrology and eventually led to a much better understanding of metamorphic conditions prevailing in an area. Several geothermobarometers have been formulated during the last 35 years for garnet-orthopyroxene-plagioclasequartz assemblage (Thomas, H., 1995, 2003, 2005, 2006 and 2008). In order to make the calculation faster, the authors have developed a program, "MPET3" for pressure-temperature calculations, using the various formulations given by different workers.

The Fe-Mg exchange reaction between garnet $(\mathrm{Gt})$ and orthopyroxene (Opx) (GOtherm) using equations given by Dhal (1980); Raith et al. (1983); Harley (1984); Sen and Bhattacharya (1984); Lee and Ganguly (1984); Perchuk et al. (1985); Lee and Ganguly (1988); Anovich and Podlesskii (1989); Perchuk and Lavrente'va (1990); Bhattacharya et al. (1991); and Lal (1993). The Fe-Mg exchange reaction of garnet-orthopyroxene is as follows:

(i) $1 / 2 \mathrm{Fe}_{2} \mathrm{Si}_{2} \mathrm{O}_{6}+1 / 3 \mathrm{Mg}_{3} \mathrm{Al}_{2} \mathrm{Si}_{3} \mathrm{O}_{12}=1 / 2 \mathrm{Mg}_{2} \mathrm{Si}_{2} \mathrm{O}_{6}+1 / 3$

$\mathrm{Fe}_{3} \mathrm{Al}_{2} \mathrm{Si}_{3} \mathrm{O}_{12}$

$1 / 2$ Ferrosilite $+1 / 3$ Pyrope $=1 / 2$ Enstatite $+1 / 3$ Almandine

To explain geobarometry properly, the authors have considered the net transfer reaction of garnet-orthopyroxeneplagioclase-quartz (GOPQ) equilibria. These mineral pairs are commonly found in the assemblages of basic granulites/ charnockite and hence are suitable for estimating equilibrium pressure of most metamorphic rocks. For this the authors have considered the equations of Newton (1978); Wells (1979); Newton and Perkins III (1982); Raith et al. (1983); Bohlen et al. (1983); Perkins and Chipera (1985); Moecher et al. 1988); Eckert et al. (1991); Bhattacharya et al. (1991); Holland and Powell (1985); calculated by Eckert et al. (1993); Berman (1988) calculated by Eckert et al. (1993); Berman (1990); calculated by Eckert et al. (1993) and Lal (1993). The equations of equilibrium are given below:

(ii) $\mathrm{Fe}_{2} \mathrm{Si}_{2} \mathrm{O}_{6}+\mathrm{CaAl}_{2} \mathrm{Si}_{2} \mathrm{O}_{8}=2 / 3 \mathrm{Fe}_{3} \mathrm{Al}_{2} \mathrm{Si}_{3} \mathrm{O}_{12}+1 / 3$ $\mathrm{Ca}_{3} \mathrm{Al}_{2} \mathrm{Si}_{3} \mathrm{O}_{12}+\mathrm{SiO}_{2}$ Ferrosilite + Anorthite $=2 / 3$ Almandine $+1 / 3$ Grossularite + Quartz

(iii) $\mathrm{Mg}_{2} \mathrm{Si}_{2} \mathrm{O}_{6}+\mathrm{CaAl}_{2} \mathrm{Si}_{2} \mathrm{O}_{8}=2 / 3 \mathrm{Mg}_{3} \mathrm{Al}_{2} \mathrm{Si}_{3} \mathrm{O}_{12}+1 / 3$ $\mathrm{Ca}_{3} \mathrm{Al}_{2} \mathrm{Si}_{3} \mathrm{O}_{12}+\mathrm{SiO}_{2}$

Enstatite + Anorthite $=2 / 3$ Pyrope $+1 / 3$ Grossularite + Quartz

The program is written in Visual Basic and the executable code of the computer program is MPET3.EXE. 


\section{GARNET-ORTHOPYROXENE EXCHANGE THERMOMETERS}

Exchange thermometers mainly imply the exchange of Fe and $\mathrm{Mg}$ between coexisting silicates. Exchange thermometers are characterized by small $\Delta \mathrm{V}$ compared to large $\Delta \mathrm{H}$ so that equilibrium constant isopleths have rather steep slope. A ratio of exchange cation in one phase divided by the same ratio in the second phase is generally taken as equilibrium constant for exchange-reactions. As an example Fe-Mg exchange between garnet and orthopyroxene is defined as $\mathrm{K}=(\mathrm{Fe} / \mathrm{Mg}) \mathrm{Gt} /(\mathrm{Fe} /$ $\mathrm{Mg}$ )Opx. This expression of partitioning of a pair of elements between two minerals is commonly referred to as distribution coefficient (KD).

This $\mathrm{Fe}-\mathrm{Mg}$ exchange equilibrium has been formulated by a number of workers by obtaining standard state thermodynamic data either by empirical method or by experiments involving the crystalline solutions (garnet and orthopyroxene) between the two cations $\mathrm{Fe}$ and $\mathrm{Mg}$. A brief description of various models is summarized as follows:

\section{Dhal (1980)}

Dhal (1980) proposed an empirical calibration for the garnetpyroxene geothermometer. The expression for geothermometer is as follows:

$\mathrm{T}(\mathrm{K})=\left[\left(1391+1509\left(\mathrm{X}_{\mathrm{Fe}}-\mathrm{X}_{\mathrm{Mg}}{ }^{\mathrm{Gt}}+2810\left(\mathrm{X}_{\mathrm{Ca}}{ }^{\mathrm{Gt}}\right)+2855\left(\mathrm{X}_{\mathrm{Mn}}^{\mathrm{Gt}}\right) /\right.\right.\right.$ $\left.\mathrm{R} \ln \mathrm{K}_{\mathrm{D}}\right]$

Where,

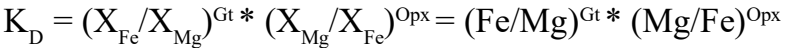

$\mathrm{i}=(\mathrm{Ca}+\mathrm{Mg}+\mathrm{Fe}+\mathrm{Mn})^{\mathrm{Gt}} ; \mathrm{X}_{\mathrm{Mg}}^{\mathrm{Gt}}=(\mathrm{Mg} / \mathrm{i})^{\mathrm{Gt}} ; \mathrm{X}_{\mathrm{Ca}}{ }^{\mathrm{Gt}}=(\mathrm{Ca} / \mathbf{i})^{\mathrm{Gt}}$;

$\mathrm{X}_{\mathrm{Mn}}{ }^{\mathrm{Gt}}=(\mathrm{Mn} / \mathrm{i})^{\mathrm{Gt}}$ and $\mathrm{X}_{\mathrm{Fe}}^{\mathrm{Gt}}=(\mathrm{Fe} / \mathrm{i})^{\mathrm{Gt}}$

$\left.\mathrm{X}_{\mathrm{Mg}}{ }^{\mathrm{Opx}}=\mathrm{Mg} / \mathrm{Mg}+\mathrm{Fe}\right) ; \mathrm{X}_{\mathrm{Fe}}{ }^{\mathrm{opx}}=\mathrm{Fe} /(\mathrm{Fe}+\mathrm{Mg})$

\section{Raith et al. (1993)}

Raith et al. (1983) proposed an empirical calibration for granulites facies rocks. The expression for geothermometer is as follows:

$\mathrm{T}(\mathrm{K})=\left[1684 /\left(\mathrm{InK}_{\mathrm{D}}+0.334\right)\right]$

Where, KD is same as defined by [4] Dahl (1980) for this as well as the subsequently discussed geothermometer.

\section{Harley (1984)}

Harley (1984) experimentally investigated the partitioning of $\mathrm{Fe}$ and $\mathrm{Mg}$ between garnet and orthopyroxene and aluminous pyroxene in the $\mathrm{P}-\mathrm{T}$ range of $5-30 \mathrm{~Kb}$ and $800-1200$ ${ }^{\circ} \mathrm{C}$ in the $\mathrm{FeO}-\mathrm{MgO}-\mathrm{Al}_{2} \mathrm{O}_{3}-\mathrm{SiO}_{2}$ (FMAS) and $\mathrm{CaO}-\mathrm{FeO}-\mathrm{MgO}-$ $\mathrm{Al}_{2} \mathrm{O}_{3}-\mathrm{SiO}_{2}$ system. Within error of the experimental data, orthopyroxene can be regarded as microscopically ideal. The effect of calcium on the Fe-Mg partitioning between garnet and orthopyroxene can be attributed to non-ideal $\mathrm{Ca}-\mathrm{Mg}$ interaction in the garnet described by interactions terms:

$\mathrm{W}_{\mathrm{Ca}-\mathrm{Mg}}^{\mathrm{Gt}}-\mathrm{W}_{\mathrm{Ca}-\mathrm{Fe}}^{\mathrm{Gt}}=1400 \pm 500 \mathrm{Cal} / \mathrm{mol}$ per site. The expression for geothermometer is as follows:
$\mathrm{T}(\mathrm{K})=\left[\left(3740+1400\left(\mathrm{X}_{\mathrm{Ca}}{ }^{\mathrm{Gt}}\right)+22.86 \mathrm{P}(\mathrm{Kb})\right) /\left(\mathrm{RlnK}_{\mathrm{D}}+1.96\right)\right]$

\section{Sen and Bhattacharya (1984)}

They formulated the orthopyroxene-garnet geothermometer for which the values of $\Delta \mathrm{H}^{\circ}, \Delta \mathrm{S}^{\circ}$ and $\Delta \mathrm{V}^{\circ}$ are:

$\Delta \mathrm{H} 1000 \mathrm{~K}=-2.713 \mathrm{~K} \mathrm{Cal} / \mathrm{mole} ; \Delta \mathrm{S} 1000 \mathrm{~K}=-0.787 \mathrm{Cal} / \mathrm{K}$ mole and $\Delta \mathrm{V} 0298 \mathrm{~K}=-0.221 \mathrm{Cal} / \mathrm{bar}$.

The expression of geothermometer is as follows:

$\mathrm{T}(\mathrm{K})=\left[\left(2713+3300\left(\mathrm{X}_{\mathrm{Ca}}{ }^{\mathrm{Gt}}\right)+195\left(\mathrm{X}_{\mathrm{Fe}}-\mathrm{X}_{\mathrm{Mg}}\right)^{\mathrm{Gt}}+0.022(\mathrm{P}-1)\right) /\right.$ $\left.\left(1.9872 * \operatorname{InK}_{\mathrm{D}}+0.787+1.5\left(\mathrm{X}_{\mathrm{Ca}}^{\mathrm{Gt}}\right)\right)\right]$

Their expression for geothermometer includes interaction parameters (W) for $\mathrm{Ca}$ as well as $\mathrm{Fe}-\mathrm{Mg}$ mixing in garnet.

\section{Lee and Ganguly (1984)}

Lee and Ganguly (1984) experimentally determined the Fe-Mg exchange for garnet-orthopyroxene pairs. Empirical adjustment form natural data in terms of $\mathrm{Ca}$ and $\mathrm{Mn}$ lead to the following expression.

$\mathrm{T}(\mathrm{K})=\left[\left(2187+1510\left(\mathrm{X}_{\mathrm{Ca}}-\mathrm{X}_{\mathrm{Mn}}\right)^{\mathrm{Gt}}+8.6 * \mathrm{P}(\mathrm{Kb})\right) /\left(\mathrm{InK}_{\mathrm{D}}+1.071\right)\right]$

\section{Perchuk et al. (1995)}

The calibrated value for $\mathrm{Fe}-\mathrm{Mg}$ exchange reaction given by [15] Perchuk et al. (1981) is as follows:

$\Delta \mathrm{H}^{\circ}{ }_{970} \mathrm{~K}=-4766 \mathrm{Cal}$.; $\Delta \mathrm{S}_{970}^{\mathrm{o}} \mathrm{K}=-2.654 \mathrm{Cal} / \mathrm{K}$ and $\Delta \mathrm{V}_{298} \mathrm{~K}$ $=-0.0234 \mathrm{Cal} / \mathrm{bar}$

Besides the non-ideal mixing of $\mathrm{Ca}$ in garnet, they also considered the interaction parameters of alumina and $\mathrm{Fe}^{+2}-\mathrm{Mg}$ mixing in orthopyroxene in their equation for geothermometer.

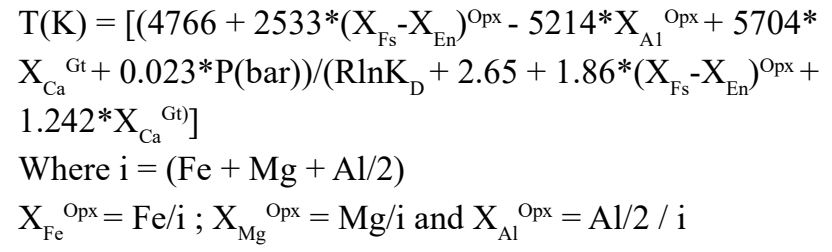

\section{Lee and Ganguly (1988)}

They refined their earlier equation proposed in 1984, through their experimental data $\mathrm{Fe}-\mathrm{Mg}$ exchange reaction between garnet and orthopyroxene.

$\mathrm{T}(\mathrm{K})=\left[\left(1981+1509.66 *\left(\mathrm{X}_{\mathrm{Ca}}-\mathrm{X}_{\mathrm{Mn}}\right)^{\mathrm{Gt}}+11.91 * \mathrm{P}(\mathrm{Kb})\right) /\left(\mathrm{InK}_{\mathrm{D}}\right.\right.$ $+0.97)]$

\section{Aranovich and Podlesskii (1989)}

They proposed an empirical calibration for garnet orthopyroxene assemblage:

$\mathrm{T}(\mathrm{K})=\left[\left((\mathrm{P}-1) * 0.02342+4766-\mathrm{A}+\left(\mathrm{X}_{\mathrm{Fe}}-\mathrm{X}_{\mathrm{Mg}}\right)^{\mathrm{Opx} * 2372-}\right.\right.$ $\left.5204 * \mathrm{X}_{\mathrm{Al}}^{\mathrm{Opx}}\right) /\left(\mathrm{RInK}_{\mathrm{D}}+2.654+\mathrm{B}+1.69 *\left(\mathrm{X}^{\mathrm{Fe}}-\mathrm{X}^{\mathrm{Mg}}\right)^{\mathrm{Opx}}\right]$ where, $\mathrm{A}=\left[-626 * \mathrm{X}_{\mathrm{Ca}}-6642 * \mathrm{X}_{\mathrm{Fe}} * \mathrm{X}_{\mathrm{Ca}}-8100 * \mathrm{X}_{\mathrm{Mg}} * \mathrm{X}_{\mathrm{Ca}}+\mathrm{X}_{\mathrm{Ca}} *\left(\mathrm{X}_{\mathrm{Mg}}\right.\right.$ $\left.\left.-\mathrm{X}_{\mathrm{Fe}}\right) * 1051.5\right]$

$\mathrm{B}=\left[1.266 * \mathrm{X}_{\mathrm{Ca}}^{2}+2.836 * \mathrm{X}_{\mathrm{Fe}} * \mathrm{X}_{\mathrm{Ca}}+3.0 * \mathrm{X}_{\mathrm{Mg}} * \mathrm{X}_{\mathrm{Ca}}+\mathrm{X}_{\mathrm{Ca}} *\left(\mathrm{X}_{\mathrm{Mg}}-\right.\right.$ $\left.\mathrm{X}_{\mathrm{Fe}} *(-0.908)\right]$ 
Geothermobarometer based on coexisting garnet-orthopyroxene-plagioclase-quartz equilibria

\section{Perchuk and Lavrente'va (1990)}

They proposed the following expression for garnet orthopyroxene geothermometer based on experimental data and thermodynamics.

$\mathrm{T}(\mathrm{K})=\left[\left(4066-347 *\left(\mathrm{X}_{\mathrm{Mg}}-\mathrm{X}_{\mathrm{Fe}}\right)^{\mathrm{OPX}}-17484 * \mathrm{X}_{\mathrm{Al}}^{\mathrm{OPX}}\right.\right.$

$\left.+5769 \mathrm{X}_{\mathrm{Ca}}^{\mathrm{Gt}}+23.42 * \mathrm{P}(\mathrm{Kb})\right) /\left(1.987 * \mathrm{InK}_{\mathrm{D}}+2.143+\right.$

$\left.\left.0.0929 *\left(\mathrm{X}_{\mathrm{Mg}}-\mathrm{X}_{\mathrm{Fe}}\right)^{\mathrm{OPX}}-12.8994 * \mathrm{X}_{\mathrm{Al}}^{\mathrm{OPX}}+3.846 * \mathrm{X}_{\mathrm{Ca}}{ }^{\mathrm{Gt}}\right)\right]$

Where $\mathrm{i}=(\mathrm{Fe}+\mathrm{Mg}+\mathrm{Al} / 2)$

$\mathrm{X}_{\mathrm{Fe}}{ }^{\mathrm{Opx}}=\mathrm{Fe} / \mathrm{i} ; \mathrm{X}_{\mathrm{Mg}}{ }^{\mathrm{Opx}}=\mathrm{Mg} / \mathrm{i}$ and $\mathrm{X}_{\mathrm{Al}}{ }^{\mathrm{Opx}}=(\mathrm{Al} / 2) / \mathrm{i}$

\section{Bhattacharya et al. (1991)}

The expression for Gt-Opx geothermometer proposed by them is as follows:

$\mathrm{T}(\mathrm{K})=\left[\left(1611+0.012(\mathrm{P}-1)+906 \mathrm{X}_{\mathrm{Ca}}^{\mathrm{Gt}}+\mathrm{A}+477\left(2 * \mathrm{X}_{\mathrm{Mg}}-1\right)\right.\right.$ Opx $\left.) /\left(\operatorname{InK}_{\mathrm{D}}+0.796\right)\right]$

The above expression can be used to estimate temperature from orthopyroxene-garnet pairs subject to the following conditions: I. Grossularite contents in garnet should be $<30$ mol \%, II. Spessartine content should be low $\left(\mathrm{X}_{\mathrm{Mn}}<0.05\right)$ such as $\mathrm{Mg} / \mathrm{Mg}<3$, III. Pressure should be less then $15 \mathrm{~Kb}$. At higher pressure the excess volume of mixing in $\mathrm{Ca}-\mathrm{Fe}$ and $\mathrm{Ca}-\mathrm{Mg}$ binaries in garnet could markedly affect the In KD-T relations:

\section{Lal (1993)}

Lal (1993) proposed the empirical calibration for garnet orthopyroxene thermo-meter. His equation is applicable for crustal rocks containing garnet with low Mn content ( $<5 \mathrm{~mol}$ $\%)$ as well as mantle derived rocks $\left(\mathrm{Cr}_{2} \mathrm{O}_{2}<5 \mathrm{wt} \%\right)$.

$\mathrm{T}(\mathrm{K})=\left[\left(3367+(\mathrm{P}-1) * 0.024-\mathrm{A}-948 *\left(\mathrm{X}_{\mathrm{Fe}}-\mathrm{X}_{\mathrm{Mg}}\right)^{\mathrm{Opx}}-1950\right.\right.$

$\left.\mathrm{X}_{\mathrm{Al}}^{\mathrm{Opx}} /\left(1.987 * \operatorname{InK}_{\mathrm{D}}+1.634+\mathrm{B}-0.34 *\left(\mathrm{X}_{\mathrm{Fe}}-\mathrm{X}_{\mathrm{Mg}}\right)^{\mathrm{Opx}}\right)\right]$

Where,

$\mathrm{A}=\left[-1256 * \mathrm{X}_{\mathrm{Mg}}{ }^{2}-2880 * \mathrm{X}_{\mathrm{Fe}}{ }^{2}+8272 *\left(\mathrm{X}_{\mathrm{Mg}} * \mathrm{X}_{\mathrm{Ca}}\right)^{\mathrm{Gt}}+\right.$

$812 * \mathrm{X}_{\mathrm{Ca}}^{\mathrm{Gt} *}\left(\mathrm{X}_{\mathrm{Mg}}-\mathrm{X}_{\mathrm{Fe}}\right)^{\mathrm{Gt}}+90 \mathrm{X}_{\mathrm{Ca}}{ }^{2}-2340 * \mathrm{X}_{\mathrm{Ca}}{ }^{\mathrm{Gt}} *\left(\mathrm{X}_{\mathrm{Fe}}+\mathrm{X}_{\mathrm{Mg}}\right)$

$\left.-3047 * \mathrm{X}_{\mathrm{Mg}} * \mathrm{X}_{\mathrm{Ca}}-1813 * \mathrm{X}_{\mathrm{Fe}} * \mathrm{X}_{\mathrm{Ca}}+\mathrm{X}_{\mathrm{Ca}} *\left(\mathrm{X}_{\mathrm{Fe}}-\mathrm{X}_{\mathrm{Mg}}\right) *(-4498)\right]$.

$\mathrm{B}=\left[1.0 * \mathrm{X}_{\mathrm{Mg}}{ }^{2}+1.7 * \mathrm{X}_{\mathrm{Fe}}^{2}-5.4 * \mathrm{X}_{\mathrm{Fe}} * \mathrm{X}_{\mathrm{Mg}}-0.35 * \mathrm{X}_{\mathrm{Ca}} *\left(\mathrm{X}_{\mathrm{Mg}}-\right.\right.$

$\left.\mathrm{X}_{\mathrm{Fe}}\right)+1.5 * \mathrm{X}_{\mathrm{Ca}}^{2}+1.666 * \mathrm{X}_{\mathrm{Ca}} *\left(\mathrm{X}_{\mathrm{Fe}}+\mathrm{X}_{\mathrm{Mg}}\right)+0.332 * \mathrm{X}_{\mathrm{Fe}} * \mathrm{X}_{\mathrm{Ca}}+$

$\left.\mathrm{X}_{\mathrm{Ca}} *\left(\mathrm{X}_{\mathrm{Fe}}-\mathrm{X}_{\mathrm{Mg}}\right) * 1.516\right]$

\section{GARNET-ORTHOPYROXENE-PLAGIOCLASE- QUARTZ EQUILIBRIA}

Vapour-absent, solid-solid, net transfer equilibria serve as potential geobarometer as they involve substantial volume change and have small positive slope in P-T space. The assemblage garnet-orthopyroxene-plagioclase-quartz in basic granulites represents a potential geobarometer. The four-phase assemblage is represented by the reaction (Thomas 1995, 2003, 2005, 2006 and 2008; Joshi et al. 1993):

$\mathrm{P}_{\mathrm{Mg}}$ Reaction: $\mathrm{CaAl}_{2} \mathrm{Si}_{2} \mathrm{O}_{8}+\mathrm{Mg}_{2} \mathrm{Si}_{2} \mathrm{O}_{6}=2 / 3 \mathrm{Mg}_{3} \mathrm{Al}_{2} \mathrm{Si}_{3} \mathrm{O}_{12}+$ $1 / 3 \mathrm{Ca}_{3} \mathrm{Al}_{2} \mathrm{Si}_{3} \mathrm{O}_{12}+\mathrm{SiO}_{2}$

Anorthite + Enstatite $=2 / 3$ Pyrope $+1 / 3$ Grossularite +
Quartz

$\mathrm{K}_{1}=\mathbf{a}_{\mathrm{Ca}}^{\mathrm{Gt} *}\left(\mathbf{a}_{\mathrm{Mg}}^{\mathrm{Gt}}\right)^{2} / \mathbf{a}_{\mathrm{An}}^{\text {plag } *} \mathbf{a}_{\mathrm{Mg}}^{\mathrm{Opx}} ; \mathrm{K}_{\mathrm{B} 1}=\mathbf{a}_{\mathrm{Ca}}^{\mathrm{Gt}} *\left(\mathbf{a}_{\mathrm{Mg}}^{\mathrm{Gt}}\right)^{2} /$

$\mathbf{a}_{\mathrm{An}}{ }^{\mathrm{plag} *}\left(\mathbf{a}_{\mathrm{Mg}}{ }^{\mathrm{Opx}}\right)^{2}$

$\mathrm{P}_{\mathrm{Fe}}$ Reaction: $\mathrm{CaAl}_{2} \mathrm{Si}_{2} \mathrm{O}_{8}+\mathrm{Fe}_{2} \mathrm{Si}_{2} \mathrm{O}_{6}=2 / 3 \mathrm{Fe}_{3} \mathrm{Al}_{2} \mathrm{Si}_{3} \mathrm{O}_{12}+1 / 3$ $\mathrm{Ca}_{3} \mathrm{Al}_{2} \mathrm{Si}_{3} \mathrm{O}_{12}+\mathrm{SiO}_{2}$

Anothite + Ferrosilite $=2 / 3$ Almandine $+1 / 3$ Grossularite + Quartz

$\mathrm{K}_{2}=\mathbf{a}_{\mathrm{Ca}}{ }^{\mathrm{Gt}} *\left(\mathbf{a}_{\mathrm{Fe}}{ }^{\mathrm{Gt}}\right)^{2} /\left(\mathbf{a}_{\mathrm{An}}{ }^{\text {plag } *} \mathbf{a}_{\mathrm{Fe}}{ }^{\mathrm{Opx}}\right) ; \mathrm{K}_{2 \mathrm{~B}}=\mathbf{a}_{\mathrm{Ca}}{ }^{\mathrm{Gt}} *\left(\mathbf{a}_{\mathrm{Fe}}{ }^{\mathrm{Gt}}\right)^{2} /\left(\mathbf{a}_{\mathrm{An}}{ }^{\text {plag } *}\right.$ $\left.\left(\mathbf{a}_{\mathrm{Fe}}^{\mathrm{Opx}}\right)^{2}\right)$

To desired barometry properly the author separated the equations given by different workers on the basis of garnet (Thomas 2006) and orthopyroxene activities:

(i) Regular Solution model for activities of garnet

$\mathbf{a}_{\mathrm{Ca}}{ }^{\mathrm{Gt}}=\mathrm{X}_{\mathrm{Ca}}{ }^{*} \exp \left[\left\{\mathrm{W}_{\mathrm{CaFe}} *\left(\mathrm{X}_{\mathrm{Fe}}{ }^{2}\right)^{\mathrm{Gt}}+\mathrm{W}_{\mathrm{CaMg}} *\left(\mathrm{X}_{\mathrm{Mg}}{ }^{2}\right)^{\mathrm{Gt}}+\left(\mathrm{W}_{\mathrm{CaFe}}+\right.\right.\right.$ $\left.\left.\left.\mathrm{W}_{\mathrm{CaMg}}-\mathrm{W}_{\mathrm{FeMg}}\right) * \mathrm{X}_{\mathrm{Fe}}^{\mathrm{Gt}} * \mathrm{X}_{\mathrm{Mg}}^{\mathrm{Gt}}\right\} / \mathrm{RT}\right]$

$\mathbf{a}_{\mathrm{Mg}}^{\mathrm{Gt}}=\mathrm{X}_{\mathrm{Mg}} * \exp \left[\left\{\mathrm{W}_{\mathrm{CaMg}} *\left(\mathrm{X}_{\mathrm{Ca}}{ }^{2}\right)^{\mathrm{Gt}}+\mathrm{W}_{\mathrm{FeMg}} *\left(\mathrm{X}_{\mathrm{Fe}}{ }^{2}\right)^{\mathrm{Gt}}+\left(\mathrm{W}_{\mathrm{CaMg}}\right.\right.\right.$ $\left.\left.\left.+\mathrm{W}_{\mathrm{FeMg}}-\mathrm{W}_{\mathrm{CaFe}}\right) * \mathrm{X}_{\mathrm{Ca}}^{\mathrm{Gt}} * \mathrm{X}_{\mathrm{Fe}}^{\mathrm{Gt}}\right\} / \mathrm{RT}\right]$

$\mathbf{a}_{\mathrm{Fe}}^{\mathrm{Gt}}=\mathrm{X}_{\mathrm{Fe}} * \exp \left[\left\{\mathrm{W}_{\mathrm{CaFe}} *\left(\mathrm{X}_{\mathrm{Ca}}{ }^{2}\right)^{\mathrm{Gt}}+\mathrm{W}_{\mathrm{FeMg}} *\left(\mathrm{X}_{\mathrm{Mg}}{ }^{2}\right)^{\mathrm{Gt}}+\left(\mathrm{W}_{\mathrm{CaFe}}+\right.\right.\right.$ $\left.\left.\left.\mathrm{W}_{\mathrm{FeMg}}-\mathrm{W}_{\mathrm{CaMg}}\right) * \mathrm{X}_{\mathrm{Ca}}^{\mathrm{Gt}} * \mathrm{X}_{\mathrm{Mg}}^{\mathrm{Gt}}\right\} / \mathrm{RT}\right]$

(ii) Regular Solution model for activities of orthopyroxene:

$\mathbf{a}_{\mathrm{Mg}}^{\mathrm{Opx}}=\mathrm{X}_{\mathrm{Mg}}{ }^{*} \exp \left[\left\{\mathrm{W}_{\mathrm{MgFe}} *\left(\mathrm{X}_{\mathrm{Fe}}^{\mathrm{Opx}}\right)^{2}+\mathrm{W}_{\mathrm{MgAl}} *\left(\mathrm{X}_{\mathrm{Al}}^{\mathrm{Opx}}\right)^{2}+\right.\right.$ $\left.\left.\left(\mathrm{W}_{\mathrm{MgFe}}+\mathrm{W}_{\mathrm{MgAl}}-\mathrm{W}_{\mathrm{FeAl}}\right) * \mathrm{X}_{\mathrm{Fe}}{ }^{\mathrm{Opx}} \mathrm{X}_{\mathrm{Al}}^{\mathrm{Opx}}\right\} / \mathrm{RT}\right]$

$\mathbf{a}_{\mathrm{Fe}}{ }^{\mathrm{Opx}}=\mathrm{X}_{\mathrm{Fe}} * \exp \left[\left\{\mathrm{W}_{\mathrm{MgFe}} *\left(\mathrm{X}_{\mathrm{Mg}}^{\mathrm{Opx}}\right)^{2}+\mathrm{W}_{\mathrm{FeAl}} *\left(\mathrm{X}_{\mathrm{Al}}{ }^{\mathrm{Opx}}\right)^{2}+\left(\mathrm{W}_{\mathrm{MgFe}}\right.\right.\right.$ $\left.\left.\left.+\mathrm{W}_{\mathrm{FeAl}}-\mathrm{W}_{\mathrm{MgAl}}\right) * \mathrm{X}_{\mathrm{Mg}}{ }^{\mathrm{Opx}} \mathrm{X}_{\mathrm{Al}}^{\mathrm{Opx}}\right\} / \mathrm{RT}\right]$

Where,

$\mathrm{X}_{\mathrm{Mg}}{ }^{\mathrm{Opx}}=\mathrm{Mg} / \mathrm{i} ; \mathrm{X}_{\mathrm{Fe}}^{\mathrm{Gt}}=\mathrm{Fe} / \mathrm{i} ; \mathrm{X}_{\mathrm{Al}}^{\mathrm{Opx}}=(\mathrm{Al} / 2) / \mathrm{i}$ and $\mathrm{i}=(\mathrm{Mg}+$ $\mathrm{Fe}+\mathrm{Al} / 2)$

(iii) Sub-regular solution model of garnet mixing by Ganguly and Saxena (1984) and Thomas (2006):

$\mathbf{a}_{\mathrm{Mg}}^{\mathrm{Gt}}=\mathrm{X}_{\mathrm{Mg}} * \exp ^{*}\left\{\left[\mathrm{X}_{\mathrm{Fe}}{ }^{2}\left[\mathrm{~W}_{\mathrm{MgFe}}+2 * \mathrm{X}_{\mathrm{Mg}} *\left(\mathrm{~W}_{\mathrm{FeMg}}-\mathrm{W}_{\mathrm{MgFe}}\right)\right]+\right.\right.$ $\mathrm{X}_{\mathrm{Ca}}{ }^{2} *\left[\mathrm{~W}_{\mathrm{MgCa}}+2 * \mathrm{X}_{\mathrm{Mg}} *\left(\mathrm{~W}_{\mathrm{CaMg}}-\mathrm{W}_{\mathrm{MgCa}}\right)\right]+\mathrm{X}_{\mathrm{Fe}} * \mathrm{X}_{\mathrm{Ca}} *[1 / 2 *$ $\left(\mathrm{W}_{\mathrm{FeMg}}+\mathrm{W}_{\mathrm{MgFe}}+\mathrm{W}_{\mathrm{CaMg}}+\mathrm{W}_{\mathrm{MgCa}}-\mathrm{W}_{\mathrm{FeCa}}-\mathrm{W}_{\mathrm{CaFe}}\right)+\mathrm{X}_{\mathrm{Mg}} *\left(\mathrm{~W}_{\mathrm{FeMg}}\right.$ $\left.-\mathrm{W}_{\mathrm{MgFe}}+\mathrm{W}_{\mathrm{CaMg}}-\mathrm{W}_{\mathrm{MgCa}}\right)+\left(\mathrm{X}_{\mathrm{Fe}}-\mathrm{X}_{\mathrm{Ca}}\right) *\left(\mathrm{~W}_{\mathrm{FeCa}}-\mathrm{W}_{\mathrm{CaFe}}\right)-(1-2 *$ $\left.\left.\left.\left.\mathrm{X}_{\mathrm{Mg}}\right) * \mathrm{C}_{123}\right]\right] / \mathrm{R} * \mathrm{~T}\right\}$

$\mathbf{a}_{\mathrm{Ca}}{ }^{\mathrm{Gt}}=\mathrm{X}_{\mathrm{Ca}}{ }^{*} \exp ^{*}\left\{\left[\mathrm{X}_{\mathrm{Mg}}{ }^{2} *\left[\mathrm{~W}_{\mathrm{CaMg}}+2 * \mathrm{X}_{\mathrm{Ca}} *\left(\mathrm{~W}_{\mathrm{MgCa}}-\mathrm{W}_{\mathrm{CaMg}}\right)\right]\right.\right.$ $+\mathrm{X}_{\mathrm{Fe}}^{2} *\left[\mathrm{~W}_{\mathrm{CaFe}}+2 * \mathrm{X}_{\mathrm{Ca}} *\left(\mathrm{~W}_{\mathrm{FeCa}}-\mathrm{W}_{\mathrm{CaFe}}\right)\right]+\mathrm{X}_{\mathrm{Mg}} * \mathrm{X}_{\mathrm{Fe}} *[1 / 2 *$ $\left(\mathrm{W}_{\mathrm{MgCa}}+\mathrm{W}_{\mathrm{CaMg}}+\mathrm{W}_{\mathrm{FeCa}}+\mathrm{W}_{\mathrm{CaFe}}-\mathrm{W}_{\mathrm{MgFe}}-\mathrm{W}_{\mathrm{FeMg}}\right)+\mathrm{X}_{\mathrm{Ca}} *\left(\mathrm{~W}_{\mathrm{MgCa}}\right.$ $\left.-\mathrm{W}_{\mathrm{CaMg}}+\mathrm{W}_{\mathrm{FeCa}}-\mathrm{W}_{\mathrm{CaFe}}\right)+\left(\mathrm{X}_{\mathrm{Mg}}-\mathrm{X}_{\mathrm{Fe}}\right) *\left(\mathrm{~W}_{\mathrm{MgFe}}-\mathrm{W}_{\mathrm{FeMg}}\right)-(1-2$ $\left.\left.\left.\left.* \mathrm{X}_{\mathrm{Ca}}\right) * \mathrm{C}_{123}\right]\right] / \mathrm{R} * \mathrm{~T}\right\}$

$\mathbf{a}_{\mathrm{Fe}}^{\mathrm{Gt}}=\mathrm{X}_{\mathrm{Fe}} * \exp *\left\{\left[\mathrm{X}_{\mathrm{Mg}}{ }^{2}\left[\mathrm{~W}_{\mathrm{FeMg}}+2 * \mathrm{X}_{\mathrm{Fe}} *\left(\mathrm{~W}_{\mathrm{MgFe}}-\mathrm{W}_{\mathrm{FeMg}}\right)\right]\right.\right.$ $+\mathrm{X}_{\mathrm{Ca}}{ }^{2} *\left[\mathrm{~W}_{\mathrm{FeCa}}+2 * \mathrm{X}_{\mathrm{Fe}} *\left(\mathrm{~W}_{\mathrm{CaFe}}-\mathrm{W}_{\mathrm{FeCa}}\right)\right]+\mathrm{X}_{\mathrm{Mg}} * \mathrm{X}_{\mathrm{Ca}} *[1 / 2 *$ $\left(\mathrm{W}_{\mathrm{MgFe}}+\mathrm{W}_{\mathrm{FeMg}}+\mathrm{W}_{\mathrm{CaFe}}+\mathrm{W}_{\mathrm{FeCa}}-\mathrm{W}_{\mathrm{MgCa}}-\mathrm{W}_{\mathrm{CaMg}}\right)+\mathrm{X}_{\mathrm{Fe}} *\left(\mathrm{~W}_{\mathrm{MgFe}}\right.$ $\left.-\mathrm{W}_{\mathrm{FeMg}}+\mathrm{W}_{\mathrm{CaFe}}-\mathrm{W}_{\mathrm{FeCa}}\right)+\left(\mathrm{X}_{\mathrm{Mg}}-\mathrm{X}_{\mathrm{Ca}}\right) *\left(\mathrm{~W}_{\mathrm{MgCa}}-\mathrm{W}_{\mathrm{CaMg}}\right)-(1-2 *$ $\left.\left.\left.\mathrm{X}_{\mathrm{Fe}}\right) * \mathrm{C}_{123}\right]\right] / \mathrm{R} * \mathrm{~T}$ \}

Where, 
$\mathrm{X}_{\mathrm{Mg}}^{\mathrm{Gt}}=\mathrm{Mg} / \mathrm{i} ; \mathrm{X}_{\mathrm{Fe}}{ }^{\mathrm{Gt}}=\mathrm{Fe} / \mathrm{i} ; \mathrm{X}_{\mathrm{Ca}}{ }^{\mathrm{Gt}}=\mathrm{Ca} / \mathrm{i}$ and $\mathrm{i}=(\mathrm{Mg}+\mathrm{Mn}+$ $\mathrm{Fe}+\mathrm{Ca})^{\mathrm{Gt}}$

\section{Equations based on regular solution models Newton (1978)}

Newton (1978) presented a barometer on the basis of experimental and thermodynamic studies and his formulation is as follows:

$\mathrm{P}_{\mathrm{Mg}}=1+\left[1370+\mathrm{T} *\left(7.03+\mathrm{RInK}_{1}\right)\right] / 0.538$

Where, $\mathrm{K}_{1}=\mathbf{a}_{\mathrm{Ca}}{ }^{\mathrm{Gt}} *\left(\mathbf{a}_{\mathrm{Mg}}{ }^{\mathrm{Gt}}\right)^{2} / \mathbf{a}_{\mathrm{An}}{ }^{\mathrm{Plag}} * \mathbf{a}_{\mathrm{Mg}}{ }^{\mathrm{Opx}}$

Newton used in his equation the activity of garnet based on regular solution mode.

Where $\mathrm{W}_{\mathrm{CaMg}}=3200 \mathrm{Cal} ; \mathrm{W}_{\mathrm{CaFe}}=0 ; \mathrm{W}_{\mathrm{MgFe}}=2500 \mathrm{Cal}$ and $\mathbf{a}_{\mathrm{An}}^{\mathrm{Opx}}=\mathrm{X}_{\mathrm{An}} * 1.28 ; \mathbf{a}_{\mathrm{Mg}}^{\mathrm{Opx}}=\mathrm{X}_{\mathrm{Mg}} \mathrm{M}_{1} * \mathrm{X}_{\mathrm{Mg}} \mathrm{M}_{2}$ (Formula based on 6 oxygen basis) and $\mathrm{X}_{\mathrm{Mg}}=\mathrm{Mg} /(\mathrm{Mg}+\mathrm{Fe})$

$\mathrm{X}_{\mathrm{Mg}}^{\mathrm{M} 1}=\left[1-\left(\mathrm{Al}^{\mathrm{vi}}+\mathrm{Fe}^{+3}+\mathrm{Ti}+\mathrm{Cr}\right)\right] * \mathrm{X}_{\mathrm{Mg}} ; \quad \mathrm{X}_{\mathrm{Mg}}{ }^{\mathrm{M} 2}=[1-(\mathrm{Mn}$

$+\mathrm{Ca}+\mathrm{Na}+\mathrm{K})]^{*} \mathrm{X}_{\mathrm{Mg}} ; \mathrm{R}=1.987 \mathrm{cal} / \mathrm{mol}$.

\section{Wells (1979)}

Wells (1979) has given an equation for barometer based on empirical studies, his equation is as follows:

$\mathrm{P}_{\mathrm{Mg}}=1+\left[3300+\mathrm{T} *\left(6.26+\mathrm{R} \ln \mathrm{K}_{1}\right)\right] / 0.56771$

Where $\mathrm{K}_{1}, \mathbf{a}_{\mathrm{An}}{ }^{\text {plag }}$ and $\mathbf{a}_{\mathrm{Mg}}{ }^{\mathrm{Opx}}$ same as given in Newton (1978) and $\mathrm{W}_{\mathrm{CaMg}}=3820 \mathrm{Cal} ; \mathrm{WCaFe}=1000 \mathrm{Cal}$ and $\mathrm{W}_{\mathrm{MgFe}}=0$ for activities of garnet.

\section{Revision by Raith et al. (1983)}

Raith et al. (1983) revised the equation given by Wells (1979), they revised on the basis of activities. They used the garnet activity same as in Newton and Perkins (1982) and aMgOpx same as in Newton (1978) and activity of anorthite in plagioclase is given below:

$\mathbf{a}_{\mathrm{An}}{ }^{\mathrm{plag}}=\mathrm{X}_{\mathrm{An}} *\left(1-\mathrm{X}_{\mathrm{An}} / 4\right) * \exp \left[\left(1-\mathrm{X}_{\mathrm{An}}\right) 2\left(2050+9392 * \mathrm{X}_{\mathrm{An}}\right)\right.$ /RT]

\section{Newton and Perkins III (1982)}

Newton and Perkins III (1982) gave thermodynamic calibration of geobarometer based on the assemblage's garnetplagioclase-orthopyroxene-quartz. The equation proposed by them is given below:

$\mathrm{P}_{\mathrm{Mg}}=1+\left[2237+\mathrm{T} *\left(7.417+\mathrm{R}_{\ln } \mathrm{K}_{1}\right)\right] / 0.567$

Where $\mathrm{K}_{1}$ is same as Newton (1978), $\mathbf{a}_{\mathrm{Mg}}{ }^{\mathrm{Opx}}$ same as in Newton (1978) model $\mathbf{a}_{\text {An }}{ }^{\text {plag }}$ same as in Wells (1979) model and activity of garnet calculated through regular solution model. They used the margulus.

$\mathrm{W}_{\mathrm{CaMg}}=3300-1.5 * \mathrm{~T}(\mathrm{~K}) \mathrm{Cal} ; \mathrm{W}_{\mathrm{CaFe}}=0 \mathrm{Cal}$ and $\mathrm{W}_{\mathrm{MgFe}}=0$

\section{Eckert et al. (1991)}

Eckert et al. (1991) investigated the $\Delta H$ of reaction and recalibrated the garnet-pyroxene-plagioclase-quartz geobarometer in the CMAS systems by solution calorimetry.
The equation proposed by them is given below: $\mathrm{P}_{\mathrm{Mg}}=1+\left[1958+\mathrm{T}^{*}\left(7.4092+\mathrm{R} \ln \mathrm{K}_{1}\right)\right] / 0.5399$ Where $\mathrm{K}_{1}, \mathrm{a}_{\mathrm{Mg}}{ }^{\mathrm{Opx}}$ and aAnplag same as Newton and Perkins III (1982).

\section{Bohlen et al. (1983)}

Bohlen et al. (1983) proposed the equation based on thermodynamics and phase equilibria. The equation proposed by them is as follows:

$\mathrm{P}_{\mathrm{Fe}}=1+\left[\mathrm{T} *\left(9.1255+\mathrm{R} \ln \mathrm{K}_{2}\right)-3705\right] / 0.6115$

Where $\mathrm{K}_{2}=\mathrm{a}_{\mathrm{Ca}}^{\mathrm{Gt}} *\left(\mathbf{a}_{\mathrm{Fe}}{ }^{\mathrm{Gt}}\right)^{2} /\left(\mathbf{a}_{\mathrm{An}}{ }^{\mathrm{Plag}} * \mathbf{a}_{\mathrm{Fe}}{ }^{\mathrm{Opx}}\right)$ and activity of garnet calculated through regular solution model:

$\mathrm{W}_{\mathrm{CaMg}}=4507.6-1.2 * \mathrm{~T}(\mathrm{~K}) \mathrm{Cal} ; \mathrm{W}_{\mathrm{CaFe}}=1377.6-1.2 * \mathrm{~T}(\mathrm{~K})$ $\mathrm{Cal}$ and $\mathrm{W}_{\mathrm{MgFe}}=3807.6-1.2 * \mathrm{~T}(\mathrm{~K}) ; \mathbf{a}_{\mathrm{An}}^{\text {plag }}=\mathrm{X}_{\mathrm{An}} * 1.28$ and $\mathrm{X}_{\mathrm{An}}=\mathrm{Ca} /(\mathrm{Ca}+\mathrm{Na}+\mathrm{K})^{\mathrm{Plag}}$

$\mathbf{a}_{\mathrm{Fe}}{ }^{\mathrm{Opx}}=\mathrm{X}_{\mathrm{Fe}} \mathrm{M}_{1} * \mathrm{X}_{\mathrm{Fe}} \mathrm{M}_{2}, \mathrm{X}_{\mathrm{Fe}} \mathrm{M}_{1}=\left[1-\left(\mathrm{Al}^{\mathrm{vi}}+\mathrm{Fe}+3+\mathrm{Ti}+\mathrm{Cr}\right]^{*}\right.$ $\left.\mathrm{X}_{\mathrm{Fe}}\right]$

$\mathrm{X}_{\mathrm{Fe}}{ }^{\mathrm{M} 2}=\left[1-(\mathrm{Mn}+\mathrm{Ca}+\mathrm{Na}+\mathrm{K}) * \mathrm{X}_{\mathrm{Fe}}\right]^{*} \mathrm{X}_{\mathrm{Fe}}$ and $\mathrm{X}_{\mathrm{Fe}}=\mathrm{Fe} /(\mathrm{Mg}$ $+\mathrm{Fe})^{\mathrm{Opx}}$

\section{Perkins and Chipera (1985)}

Perkins and Chipera (1985) gave an empirical calibration for garnet-orthopyroxene -plagioclase-quartz barometry. They calculated the activity of garnet through sub-regular mixing in garnet at $750^{\circ} \mathrm{C}$ using equation given by Ganguly and Saxena, (1984). They solution used the following margulus parameters for activity:

$\mathrm{W}_{\mathrm{FeMg}}=200 \mathrm{Cal} ; \mathrm{W}_{\mathrm{MgFe}}=2500 ; \mathrm{W}_{\mathrm{CaMg}}=2515.5 ; \mathrm{W}_{\mathrm{MgCa}}=$ -534.5; $\mathrm{W}_{\mathrm{CaFe}}=-2164.5 ; \mathrm{W}_{\mathrm{FeCa}}=3085.5$ and $\mathrm{C} 123=0, \mathbf{a}_{\mathrm{Mg}}^{\mathrm{Opx}}$ as in Newton (1978) and $\mathbf{a}_{\mathrm{Fe}}{ }^{\mathrm{Opx}}$ as in Bohlen et al. (1983).

The proposed equation is as follows:

$\mathrm{P}_{\mathrm{Mg}}=1+\left[\mathrm{T}^{*}\left(7.335+\mathrm{R} \ln \mathrm{K}_{1}\right)+1310\right] / 0.5408$

Where $\mathrm{K}_{1}=\mathbf{a}_{\mathrm{Ca}}{ }^{\mathrm{Gt}} *\left(\mathbf{a}_{\mathrm{Mg}}{ }^{\mathrm{Gt}}\right)^{2} / \mathbf{a}_{\mathrm{An}}{ }^{\mathrm{Plag}} * \mathbf{a}_{\mathrm{Mg}}{ }^{\mathrm{Opx}}$

$\mathrm{P}_{\mathrm{Fe}}=1+\left[\mathrm{T}^{*}(8.364+\mathrm{R} \operatorname{lnK} 2)-2247\right] / 0.5849$

Where $\mathrm{K} 2=\mathbf{a}_{\mathrm{Ca}}{ }^{\mathrm{Gt}} *\left(\mathbf{a}_{\mathrm{Fe}}{ }^{\mathrm{Gt}}\right)^{2} /\left(\mathbf{a}_{\mathrm{An}}{ }^{\mathrm{Plag}} * \mathbf{a}_{\mathrm{Fe}}{ }^{\mathrm{Opx}}\right)$

and $\mathbf{a}_{\mathrm{An}}{ }^{\mathrm{plag}}=\mathrm{X}_{\mathrm{An}}\left(1-\mathrm{X}_{\mathrm{An}}\right)^{2} / 4 * \exp \left[\left(1-\mathrm{X}_{\mathrm{An}}\right)^{2} *(2050+9392 *\right.$ $\left.\left.\mathrm{X}_{\mathrm{An}}\right) /(1.987 * 1023)\right]$

\section{Moecher et al. (1988)}

Moecher et al. (1988) proposed the empirical calibration for pyroxene-garnet-plagioclase-quartz geobarometer. They calculated the activity of garnet through subregular solution mixing in garnet using equation of Ganguly and Saxena (1984). $\mathbf{a}_{\mathrm{Mg}}{ }^{\mathrm{Opx}}$ as in Newton (1978), $\mathbf{a}_{\mathrm{Fe}}{ }^{\mathrm{Opx}}$ as in Bohlen et al. (1983) and $\mathbf{a}_{\text {An }}{ }^{\text {Plag }}$ same as in wells (1979). They used the margulus parameters for garnet activity which is as follows:

$\mathrm{W}_{\mathrm{FeMg}}=200 \mathrm{Cal} ; \mathrm{W}_{\mathrm{MgFe}}=2500 ; \mathrm{W}_{\mathrm{CaMg}}=4047-1.5 * \mathrm{~T} ; \mathrm{W}_{\mathrm{MgCa}}=$ $1000-1.5^{*} \mathrm{~T} ; \mathrm{W}_{\mathrm{CaFe}}=150-1.5 * \mathrm{~T} ; \mathrm{W}_{\mathrm{FeCa}}=4038-1.5^{*} \mathrm{~T}$ and $\mathrm{C}_{123}$ $=-4640$

The equation given by them is as follows: 
$\mathrm{P}_{\mathrm{Fe}}=1+\left[\mathrm{T} *\left(9.353+\mathrm{R} \ln \mathrm{K}_{2}\right)-3244\right] / 0.61233$

Where $\mathrm{K}_{2}=\mathbf{a}_{\mathrm{Ca}}^{\mathrm{Gt}} *\left(\mathbf{a}_{\mathrm{Fe}}{ }^{\mathrm{Gt}}\right)^{2} / \mathbf{a}_{\mathrm{An}}{ }^{\mathrm{Plag}} * \mathbf{a}_{\mathrm{Fe}}{ }^{\mathrm{Opx}}$

\section{Aranovich and Podlesskii (1989)}

Aranovich and Podlesskii (1989) gave the equation for simultaneously operating reactions. They calculated the activity of garnet through sub-regular solution of mixing in garnet using equation of Ganguly and Saxena (1989). They used the margulus parameter for activity of garnet as follows:

$\mathrm{W}_{\mathrm{FeMg}}=0 \mathrm{Cal} ; \mathrm{W}_{\mathrm{MgFe}}=0 ; \mathrm{W}_{\mathrm{CaMg}}=4050-1.5 * \mathrm{~T} ; \mathrm{W}_{\mathrm{MgCa}}=$

$1000-1.5^{*} \mathrm{~T}$

$\mathrm{W}_{\mathrm{CaFe}}=-3321+1.481 * \mathrm{~T} ; \mathrm{W}_{\mathrm{FeCa}}=374-0.234 * \mathrm{~T}$ and $\mathrm{C}_{123}=$ 0 and calculated the activity of $\mathrm{Mg}$ in $\mathrm{Opx}\left(\mathbf{a}_{\mathrm{Mg}}{ }^{\mathrm{Opx}}\right)$, through regular solution model of orthopyroxene mixing model.

Where $\mathrm{W}_{\mathrm{MgFe}}=-2372+1.69 * \mathrm{~T} ; \mathrm{W}_{\mathrm{FeAl}}=-6441$ and $\mathrm{W}_{\mathrm{MgAl}}=$ $-1237$.

The proposed equation is given below:

$\mathrm{P}_{\mathrm{Mg}}=1+\left[522+\mathrm{T}^{*}(8.794+\mathrm{R} \ln \mathrm{K} 1 \mathrm{~B}) /(0.56156+\right.$

$\left.0.285 *\left(\mathrm{X}_{\mathrm{Al}}{ }^{\mathrm{Opx}}\right)^{2}-1 / 3 *\left[\left(0.075-4.566 *\left(0.2-\mathrm{X}_{\mathrm{Ca}}{ }^{\mathrm{Gt}}\right)^{2}\right)\right]\right)$

Where ,

$\mathrm{X}_{\mathrm{Al}}^{\mathrm{Opx}}=(\mathrm{Al} / 2) /\left[(\mathrm{Al} / 2)+\mathrm{Mg}+\mathrm{Fe}^{2}\right]$

$\mathbf{a}_{\mathrm{An}}{ }^{\text {plag }}=\mathrm{X}_{\mathrm{An}} * \exp \left\{\left(1-\mathrm{X}_{\mathrm{Ca}}\right)^{2} *\left[\mathrm{~W}_{\mathrm{CaNa}}+2 * \mathrm{X}_{\mathrm{Ca}} *\left(\mathrm{~W}_{\mathrm{NaCa}}-\right.\right.\right.$

$\left.\left.\left.\mathrm{W}_{\mathrm{CaNa}}\right)\right]\right\} /(\mathrm{R} * \mathrm{~T})$

$\mathrm{W}_{\mathrm{CaFe}}=3321+1481 * \mathrm{~T} ; \mathrm{W}_{\mathrm{FeCa}}=374-0.234 * \mathrm{~T}$ and $\mathrm{C}_{123}=0$;

$\mathrm{W}_{\mathrm{CaNa}}=1980-1.526 * \mathrm{~T} \mathrm{Cal}$;

$\mathrm{W}_{\mathrm{NaCa}}=6860-3.784 * \mathrm{~T} \mathrm{Cal}$

\section{Bhattacharya et al. (1991)}

Bhattacharya et al. (1991) gave an improved set of a-X parameters for $\mathrm{Fe}-\mathrm{Mg}-\mathrm{Ca}$ garnets and refinements of the orthopyroxene-garnet-plagioclase-quartz barometer. They calculated the activity of garnet through sub-regular solution mixing in garnet at $1000 \mathrm{~K}$ using equation of Ganguly and Saxena (1984) and aAnPlag at $750^{\circ} \mathrm{C}$ as given in Perkins and Chipera (1985). They used the margulus values for garnet activity as follows:

$\mathrm{W}_{\mathrm{FeMg}}=270 \mathrm{Cal} ; \mathrm{W}_{\mathrm{MgFe}}=1482 ; \mathrm{W}_{\mathrm{CaMg}}=1800 ; \mathrm{W}_{\mathrm{MgCa}}=1800 ;$ $\mathrm{W}_{\mathrm{CaFe}}=0 ; \mathrm{W}_{\mathrm{FeCa}}=0$ and $\mathrm{C}_{123}=0$

$\mathrm{P}_{\mathrm{Mg}}=1+\left[\mathrm{T}^{*}\left(7.417+\mathrm{R} \ln \mathrm{K}_{1 \mathrm{~B}}\right)+2237\right] / 0.567$

Where $\mathrm{K}_{\mathrm{lB}}=\mathbf{a}_{\mathrm{Ca}}{ }_{\mathrm{Gt}} *\left(\mathbf{a}_{\mathrm{Mg}}{ }^{\mathrm{Gt}}\right)^{2} /\left(\mathbf{a}_{\mathrm{An}}{ }^{\text {Plag }} *\left(\mathbf{a}_{\mathrm{Mg}}{ }^{\mathrm{Opx}}\right)^{2}\right.$

$\mathrm{P}_{\mathrm{Fe}}=1+\left[\mathrm{T}^{*}\left(8.153+\mathrm{R} \ln \mathrm{K}_{2 \mathrm{~B}}\right)-2153\right] / 0.5928$

Where $\mathrm{K}_{2 \mathrm{~B}}=\mathbf{a}_{\mathrm{Ca}}{ }^{\mathrm{Gt}} *\left(\mathbf{a}_{\mathrm{Fe}}{ }^{\mathrm{Gt}}\right)^{2} /\left(\mathbf{a}_{\mathrm{An}}^{\mathrm{Plag} *}\left(\mathbf{a}_{\mathrm{Fe}}^{\mathrm{Opx}}\right)^{2}\right.$

$\mathbf{a}_{\mathrm{Mg}}^{\mathrm{Opx}}=\mathrm{X}_{\mathrm{Mg}} * \exp \left[984 *\left(\mathrm{X}_{\mathrm{Fe}}{ }^{\mathrm{Opx}}\right)^{2} /(1.987 * 1000)\right]$ and

$\mathbf{a}_{\mathrm{Fe}}{ }^{\mathrm{Opx}}=\mathrm{X}_{\mathrm{Fe}} * \exp \left[984 *\left(\mathrm{X}_{\mathrm{Mg}}^{\mathrm{Opx}}\right)^{2} /(1.987 * 1000)\right]$

\section{Lal (1993)}

Lal (1993) gave an internally consistent recalibrations of mineral equilibria for geothermobarometry involving garnetorthopyroxene-plagioclase-quartz assemblages. He calculated the activity of garnet through sub-regular solution mixing in garnet using equation of Ganguly and Saxena (1984), where he used the margulus values as follows:

$\mathrm{W}_{\mathrm{FeMg}}=-1256+1.0 * \mathrm{~T} ; \mathrm{W}_{\mathrm{MgFe}}=2880-1.7 * \mathrm{~T} ; \mathrm{W}_{\mathrm{CaMg}}=4047$

$-1.5 * \mathrm{~T} ; \mathrm{W}_{\mathrm{MgCa}}=1000-1.5 * \mathrm{~T} ; \mathrm{W}_{\mathrm{CaFe}}=-723+0.332 * \mathrm{~T} ; \mathrm{W}_{\mathrm{FeCa}}$ $=1090$ and $\mathrm{C}_{123}=-4498+1.516^{*} \mathrm{~T}$,

Activity of $\mathrm{Mg}$ and $\mathrm{Fe}$ in $\mathrm{Opx}\left(\mathbf{a}_{\mathrm{Mg}}{ }^{\mathrm{Opx}}\right.$ and $\left.\mathbf{a}_{\mathrm{Fe}}{ }^{\mathrm{Opx}}\right)$ calculated through regular solution model of orthopyroxene mixing model,

Where; $\mathrm{W}_{\mathrm{MgFe}}=948-0.34 * \mathrm{~T} ; \mathrm{W}_{\mathrm{FeAl}}=-1950$ and $\mathrm{W}_{\mathrm{MgAl}}=$ 0 and activity of anorthite in plagioclase $\left(\mathbf{a}_{\mathrm{An}}{ }^{\text {plag }}\right)$ same as in Wells (1979) see model 2b. The equation proposed by him is given below:

$\mathrm{P}_{\mathrm{Mg}}=1+\left[\mathrm{T} *\left(5.376+\mathrm{R} \ln \mathrm{K}_{2}\right)+3985\right] / 0.5614$
$\mathrm{P}_{\mathrm{Fe}}=1+\left[\mathrm{T} *\left(8.644+\mathrm{R} \ln \mathrm{K}_{2 \mathrm{~B}}\right)+2749\right] / 0.60946$

Holland and Powell (1985) calculated by Eckert et al. (1993)

Holland and Powell (1985) gave an internally consistent thermodynamic data set with uncertainties and correlations and Eckert et al. (1993) recalibrated based on thermo-chemically.

$\mathrm{P}_{\mathrm{Mg}}=1+\left[\mathrm{T}^{*}\left(6.525+\mathrm{R}_{\ln \mathrm{K}_{1}}\right)+2632\right] / 0.5399$

Where $\mathbf{a}_{\mathrm{Ca}}{ }^{\mathrm{Gt}} ; \mathbf{a}_{\mathrm{Mg}}{ }_{\mathrm{Gt}} ; \mathbf{a}_{\mathrm{An}}{ }^{\mathrm{Plag}}$ and $\mathbf{a}_{\mathrm{Mg}}{ }^{\mathrm{Opx}}$ same as in Newton and Perkins (1982).

Holland and Powell (1990) calculated by Eckert et al. (1993)

Holland and Powell (1990) gave an enlarged and updated internally consistent thermodynamic data set with uncertainties and correlations: the system $\mathrm{K}_{2} \mathrm{O}-\mathrm{Na}_{2} \mathrm{O}-\mathrm{CaO}-\mathrm{MgO}-\mathrm{MnO}-$ $\mathrm{FeO}-\mathrm{Fe}_{2} \mathrm{O}_{3}-\mathrm{Al}_{2} \mathrm{O}_{3}-\mathrm{TiO}_{2}-\mathrm{SiO}_{2}-\mathrm{C}-\mathrm{H}_{2}-\mathrm{O}_{2}$ and Eckert et al. (1993) recalibrated thermochemically.

$\mathrm{P}_{\mathrm{Mg}}=1+[\mathrm{T} *(6.931+\mathrm{R} \ln \mathrm{K} 1)+2513] / 0.5478$

Where $\mathbf{a}_{\mathrm{Ca}}^{\mathrm{Gt}} ; \mathbf{a}_{\mathrm{Mg}}^{\mathrm{Gt}} ; \mathbf{a}_{\mathrm{An}}{ }^{\text {plag }}$ and $\mathbf{a}_{\mathrm{Mg}}{ }^{\mathrm{Opx}}$ same as in Newton and Perkins (1982).

\section{Berman (1988) calculated by Eckert et al. (1993)}

Berman (1988) gave an internally-consistent thermodynamic data for minerals in the system $\mathrm{NaO}_{2}-\mathrm{K}_{2} \mathrm{O}-\mathrm{CaO}-\mathrm{MgO}-\mathrm{FeO}$ $-\mathrm{Fe}_{2} \mathrm{O}_{3}-\mathrm{Al}_{2} \mathrm{O}_{3}-\mathrm{SiO}_{2}-\mathrm{H}_{2} \mathrm{O}-\mathrm{CO}_{2}$ and also calculated the activity of garnet using $\mathrm{Ca}-\mathrm{Fe}-\mathrm{Mg}$ ternary sub-regular solution of mixing in garnet (Berman 1990) calculated at constant pressure of $7 \mathrm{Kbar}$ equation is given below, and Eckert et al. (1993) recalibrated the Berman equation thermochemically.

$\mathrm{PMg}=1+\left[\mathrm{T} *\left(7.459+\mathrm{R} \ln \mathrm{K}_{1}\right)+1269\right] / 0.54995$

Where $\mathrm{a}_{\mathrm{An}}{ }^{\text {Plag }}, \mathrm{a}_{\mathrm{Mg}}{ }^{\text {Opx }}$ same as in Newton and Perkins (1982) and

$\mathbf{a}_{\mathrm{Mg}}{ }^{\mathrm{Gt}}=\mathrm{X}_{\mathrm{Mg}} * \exp *\left\{\left[\mathrm{~W}_{112}\left(\mathrm{X}_{\mathrm{Ca}}{ }^{2}-2 * \mathrm{X}_{\mathrm{Ca}}{ }^{2} * \mathrm{X}_{\mathrm{Mg}}\right)+\mathrm{W}_{112} *(2 *\right.\right.$

$\left.\mathrm{X}_{\mathrm{Ca}} * \mathrm{X}_{\mathrm{Mg}}-2 * \mathrm{X}_{\mathrm{Ca}} * \mathrm{X}_{\mathrm{Mg}}{ }^{2}\right)+\mathrm{W}_{113} *\left(-2 * \mathrm{X}_{\mathrm{Ca}}{ }^{2} * \mathrm{X}_{\mathrm{Fe}}\right)+\mathrm{W}_{133} *(-2$

$\left.* \mathrm{X}_{\mathrm{Ca}} * \mathrm{X}_{\mathrm{Fe}}{ }^{2}\right)+\mathrm{W} 223 *\left(2 * \mathrm{X}_{\mathrm{Mg}} * \mathrm{X}_{\mathrm{Fe}}-2 * \mathrm{X}_{\mathrm{Mg}}{ }^{2} * \mathrm{X}_{\mathrm{Fe}}\right)+\mathrm{W}_{233}$

$*\left(\mathrm{X}_{\mathrm{Fe}}{ }^{2}-2 * \mathrm{X}_{\mathrm{Mg}} * \mathrm{X}_{\mathrm{Fe}}{ }^{2}\right)+\mathrm{W}_{123} *\left(\mathrm{X}_{\mathrm{Ca}} * \mathrm{X}_{\mathrm{Fe}}-2 * \mathrm{X}_{\mathrm{Ca}} \mathrm{X}_{\mathrm{Mg}} *\right.$ $\left.\left.\mathrm{X}_{\mathrm{Fe}}\right) /(\mathrm{R} * \mathrm{~T})\right\}$

$\mathbf{a}_{\mathrm{Ca}}{ }^{\mathrm{Gt}}=\mathrm{X}_{\mathrm{Ca}} * \exp *\left\{\left[\mathrm{~W}_{112}\left(2 * \mathrm{X}_{\mathrm{Ca}} * \mathrm{X}_{\mathrm{Mg}}-2 * \mathrm{X}_{\mathrm{Ca}}{ }^{2} * \mathrm{X}_{\mathrm{Mg}}\right)+\mathrm{W}_{122}\right.\right.$ $*\left(\mathrm{X}_{\mathrm{Mg}}^{2}-2 * \mathrm{X}_{\mathrm{Ca}} * \mathrm{X}_{\mathrm{Ca}} * \mathrm{X}_{\mathrm{Mg}}^{2}\right)+\mathrm{W}_{113} *\left(2 * \mathrm{X}_{\mathrm{Ca}} * \mathrm{X}_{\mathrm{Fe}}-2 * \mathrm{X}_{\mathrm{Fe}} *\right.$ 
Table 1: Electron micro probe analyses $(\mathrm{Wt} \%)$ and structural formula of garnet and orthopyroxene in basic granulite from Thana, District Bhilwara, Rajasthan. (Source: Thomas 1995).

\begin{tabular}{|c|c|c|c|}
\hline Oxides & $\begin{array}{l}\text { Garnet Sample No H90/2, } \\
\text { Point } 10\end{array}$ & $\begin{array}{l}\text { Orthopyroxene Sample No } \\
\text { H90/2, Point } 08\end{array}$ & $\begin{array}{l}\text { Plagioclase Sample No. H90/2 } \\
\text { Point }\end{array}$ \\
\hline $\mathrm{SiO}_{2}$ & 37.926 & 51.408 & 56.539 \\
\hline $\mathrm{TiO}_{2}$ & 0.003 & 0.254 & 0.000 \\
\hline $\mathrm{Al}_{2} \mathrm{O}_{3}$ & 21.208 & 1.116 & 27.461 \\
\hline $\mathrm{Cr}_{2} \mathrm{O}_{3}$ & 0.016 & 0.063 & 0.000 \\
\hline $\mathrm{Fe}_{2} \mathrm{O}_{3}$ & 0.678 & 0.418 & 0.000 \\
\hline $\mathrm{FeO}$ & 26.927 & 28.285 & 0.081 \\
\hline $\mathrm{MnO}$ & 1.344 & 0.499 & 0.000 \\
\hline $\mathrm{MgO}$ & 4.319 & 17.499 & 0.000 \\
\hline $\mathrm{CaO}$ & 7.970 & 1.161 & 10.165 \\
\hline $\mathrm{Na}_{2} \mathrm{O}$ & 0.000 & 0.051 & 5.826 \\
\hline $\mathrm{K}_{2} \mathrm{O}$ & 0.000 & 0.000 & 0.135 \\
\hline TOTAL & 100.389 & 100.754 & 100.207 \\
\hline Cation & Cation & Cation & Cation \\
\hline $\mathrm{Si}$ & 5.9583 & 1.9625 & 2.5365 \\
\hline $\mathrm{Ti}$ & 0.0003 & 0.0073 & 0.0000 \\
\hline $\mathrm{Al}$ & 3.9269 & 0.0502 & 1.4520 \\
\hline $\mathrm{Cr}$ & 0.0020 & 0.0019 & 0.0000 \\
\hline $\mathrm{Fe}^{+3}$ & 0.0801 & 0.0120 & 0.0000 \\
\hline $\mathrm{Mg}$ & 1.0113 & 0.9957 & 0.0000 \\
\hline $\mathrm{Ca}$ & 1.3415 & 0.0475 & 0.4886 \\
\hline $\mathrm{Mn}$ & 0.1788 & 0.0162 & 0.0000 \\
\hline $\mathrm{Fe}$ & 3.5378 & 0.9030 & 0.0031 \\
\hline $\mathrm{Na}$ & 0.0000 & 0.0037 & 0.5068 \\
\hline $\mathrm{K}$ & 0.0000 & 0.0000 & 0.0077 \\
\hline
\end{tabular}

Table 2: Temperature $\left(\right.$ in $^{\circ} \mathrm{C}$ ) calculated at 7000 bars from garnet-orthopyroxene thermometer for the assemblages of basic granulites (H90/2) from Shivpura area, District Bhilwara, Rajasthan.

\begin{tabular}{|c|c|c|c|c|c|}
\hline S. No. & \multicolumn{2}{|l|}{ Authors } & KD & InK $_{\mathrm{D}}$ & Temperature $^{\circ} \mathrm{C}$ \\
\hline \multicolumn{6}{|c|}{$1 / 2$ Ferrosilite $+1 / 3$ Pyrope $=1 / 2$ Enstatite $+1 / 3$ Almandine } \\
\hline 1. & \multicolumn{2}{|c|}{ DAHL (1980) } & 3.8574 & 1.3500 & 742 \\
\hline 2. & \multicolumn{2}{|c|}{ RAITH et al. (1983) } & 3.8574 & 1.3500 & 705 \\
\hline 3. & \multicolumn{2}{|c|}{ HARLEY (1984) } & 3.8574 & 1.3500 & 634 \\
\hline 4. & \multicolumn{2}{|c|}{ SEN and BHATTACHARYA (1984) } & 3.8574 & 1.3500 & 694 \\
\hline 5. & \multicolumn{2}{|c|}{ LEE and GANGULY (1984) } & 3.8574 & 1.3500 & 811 \\
\hline 6. & \multicolumn{2}{|c|}{ PERCHUK et al. (1985) } & 3.8574 & 1.3500 & 814 \\
\hline 7. & \multicolumn{2}{|c|}{ LEE and GANGULY (1988) } & 3.8574 & 1.3500 & 780 \\
\hline 8. & \multicolumn{2}{|c|}{ ARANOVICH and PODLESSKII (1989) } & 3.8574 & 1.3500 & 753 \\
\hline 9. & \multicolumn{2}{|c|}{ PERCHUK and LAVRENTE'VA (1990) } & 3.8574 & 1.3500 & 681 \\
\hline 10. & \multicolumn{2}{|c|}{ BHATTACHARYA et al. (1991) } & 3.8574 & 1.3500 & 698 \\
\hline 11. & \multicolumn{2}{|l|}{ LAL (1993) } & 3.8574 & 1.3500 & 728 \\
\hline & $\mathrm{XFe}(\mathrm{OPX})$ & $\mathrm{XMg}(\mathrm{OPX})$ & $\mathrm{XAl}(\mathrm{OPX})$ & & \\
\hline & 0.469384 & 0.517569 & 0.013047 & & \\
\hline & $\mathrm{XFe}(\mathrm{Gt})$ & $\mathrm{XMg}(\mathrm{Gt})$ & $\mathrm{XCa}(\mathrm{Gt})$ & XMn (Gt) & \\
\hline & 0.582891 & 0.166623 & 0.221027 & 0.029459 & \\
\hline
\end{tabular}


Table 3: Pressure in $(\mathrm{Kb})$ calculated at $1023 \mathrm{~K}$ from garnet-orthopyroxene-plagioclase-quartz barometer for the assemblages of granulites (H90/2) from Shivpura area, District Bhilwara, Rajasthan (Thomas 2005).

\begin{tabular}{|c|c|c|}
\hline Author & $\operatorname{lnK}_{\mathrm{D}}$ & Pressure (Kb) Calculated for $P_{\mathrm{Mg}}$ Reaction \\
\hline \multicolumn{3}{|c|}{ Anorthite + Enstatite $=1 / 3$ Grossularite $+2 / 3$ Pyrope + Quartz } \\
\hline Newton (1978) & -1.436597 & 10.49 \\
\hline Wells (1979) & -2.23514 & 9.9 \\
\hline Raith et al. (1983) & -2.826595 & 6.97 \\
\hline Newton and Perkins III (1982) & -2.826595 & 7.19 \\
\hline Eckert et al. (1991) & -2.826595 & 7.02 \\
\hline Perkins and Chipera (1985) & -2.102059 & 8.36 \\
\hline Holland and Powell, (1985) calculated by Eckert et al. (1993) & -2.826595 & 6.6 \\
\hline Holland and Powell, (1990) calculated by Eckert et al. (1993) & -2.826595 & 7.64 \\
\hline Berman (1988) calculated by Eckert et al. (1993) & -2.511311 & 6.9 \\
\hline Aranovich and Podlesskii (1989) & 3.061691 & 4.3 \\
\hline Bhattacharya et al. (1991) & 2.740128 & 7.5 \\
\hline Lal (1993) & 2.58783 & 7.53 \\
\hline Author & $\ln K_{\mathrm{p}}$ & Pressure $(\mathrm{Kb})$ Calculated for $\mathrm{P}_{\mathrm{Fe}}$ Reaction \\
\hline \multicolumn{3}{|c|}{ Anorthite + Ferrosilite $=1 / 3$ Grossularite $+2 / 3$ Almandine + Quartz } \\
\hline Bohlen et al. (1983) & -0.357622 & 8.02 \\
\hline Perkins and Cipera (1985) & -0.613129 & 8.66 \\
\hline Moecher et al. (1988) & -0.412138 & 8.96 \\
\hline Bhattacharya et al. (1991) & -0.825801 & 7.64 \\
\hline Lal (1993) & -0.387665 & 8.71 \\
\hline
\end{tabular}

$\left.\mathrm{X}_{\mathrm{Ca}}^{2}\right)+\mathrm{W}_{133} *\left(\mathrm{X}_{\mathrm{Fe}}^{2}-2 * \mathrm{X}_{\mathrm{Ca}} * \mathrm{X}_{\mathrm{Fe}}^{2}\right)+\mathrm{W}_{223} *\left(-2 * \mathrm{X}_{\mathrm{Mg}}^{2} * \mathrm{X}_{\mathrm{Fe}}\right.$ )$\left.+\mathrm{W}_{223} *\left(-2 * \mathrm{X}_{\mathrm{Fe}}^{2} * \mathrm{X}_{\mathrm{Mg}}\right)+\mathrm{W}_{123} *\left(\mathrm{X}_{\mathrm{Mg}} \mathrm{X}_{\mathrm{Fe}}-2 * \mathrm{X}_{\mathrm{Ca}} * \mathrm{X}_{\mathrm{Mg}} \mathrm{X}_{\mathrm{Fe}}\right)\right]$ / $\mathrm{R} * \mathrm{~T}\}$

Where, $\quad \mathrm{W}_{112}=1774-1.5 * \mathrm{~T} ; \mathrm{W}_{122}=5569-1.5 * \mathrm{~T} ; \mathrm{W}_{113}$ $=1714-0.405 * \mathrm{~T} ; \mathrm{W}_{133}=259-0.405 * \mathrm{~T} ; \mathrm{W}_{223}=24 ; \mathrm{W}_{233}=$ $329 ; \mathrm{W}_{123}=4834-1.9017 * \mathrm{~T}$.

\section{PROGRAM DESCRIPTION}

The software consists of three programs as it is clear from; it prompts the user for the choice of:

1. Gt-Opx exchange reaction (GOTherm)

2. Gt-Opx-Plag-Qz equilibria (GOPQ)

3. Exit

If the temperature calculation is intended through (GOTherm) garnet-orthopyroxene exchange reaction enter the option No. 1. It automatically proceeds into the program (GOtherm) for calculation. Besides this, GOTherm also consists of three subprograms. On running, it prompts the user three choices, viz., ENTER DATA; CALCULATE and DISPLAY RESULTS and EXIT from GOtherm.

The first choice is ENTER DATA when opted asks for file name. The file selected is opened and displayed on the screen.
If the file asked for does not exist then a file by the asked name is automatically created. The program then asks for data. The data requirement is in the form of structural formula units or atomic formula units (a.f.u.) of $\mathrm{Fe}, \mathrm{Mn}, \mathrm{Mg}, \mathrm{Ca}$ for garnet and $\mathrm{Fe}, \mathrm{Mg}, \mathrm{Mn}, \mathrm{Ca}, \mathrm{Al}$ and $\mathrm{Na}$ for orthopyroxene. Pressure (in bars) at which the temperature is to be calculated is also required. The data entry operation can be terminated by entering 0 (zero) at the S. No. prompt.

The second choice, CALCULATED AND DISPLAY RESULTS however requires the data file name. The program first displays the data file contents and then proceeds for calculation of temperature through different models. This program may be looked upon as an operational block, which performs the specific operations on the data to give output in a fixed format (Table 2).

The third option is for termination of the program and return to the main program. In a similar way, if the user wishes to calculate pressure through garnet orthopyroxene -plagioclasequartz equilibria (GOPQ), option no.2 is too entered. The program automatically enters into the subprogram (GOPQ) for pressure calculation. It also works like GOTherm. The data requirement in this program is also in the form structural formula units or atomic formula unit (a.f.u.) of $\mathrm{Fe}, \mathrm{Mn}, \mathrm{Mg}, \mathrm{Ca}$ for garnet, $\mathrm{Ca}, \mathrm{Na}, \mathrm{K}$ for plagioclase and $\mathrm{Fe}, \mathrm{Mg}, \mathrm{Mn}, \mathrm{Ca}, \mathrm{Aliv}$, 
Alvi, Fe3, Cr, Ti, K and $\mathrm{Na}$ for orthopyroxene.

Temperature (in K) at which the pressure is to be calculated is also required. The data ENTRY operation can be terminated by entering 0 (zero) at the S. No. prompts. Rest other options work like GOTherm subprogram.

\section{PROGRAM VALIDATION}

To validate the program, several data had been manually calculated (Thomas 1995, 2005; Joshi et. al. 1993, Thomas 2006 and 2008) and reprocessed. It was observed that all the results are same as manually calculated. For example, one set of data is given in (Table 1) and the results obtained are shown in (Table 2 and 3). Through there is some variation among the results obtained from individual models. This discrepancy might be due to that fact that some of the models are based on empirical studies, some on thermodynamically and experimental work. Obviously, the experimentally calibrated models should be the preferred geothermobarometers. But according to author's observation garnet -orthopyroxene $\mathrm{Fe}-\mathrm{Mg}$ exchange thermometer and garnet -orthopyroxene -plagioclase -quartz barometer of Bhattacharya et al. (1991) and Lal (1993) yield temperature and pressure estimates which agree with independent petrologic temperature constraints. These models take into accounts non-ideal Fe-Mg mixing in orthopyroxene and should be preferred over the other models, since it shows very little compositional dependence.

\section{ACKNOWLEDGEMENT}

The author (HT) is thankful to Head, Department of Applied Geology, for providing necessary facilities to carry out this research work.

\section{REFERENCES}

Aranovich, L. Ya. and Podlesskii, K. K., 1989, Geothermobarometry of high grade metapelites: simultaneously operating reactions. In: Evolution of Metamorphic belts (eds. Daly, J. S., Cliff, R. A. and Yardley, B. W. D.) Geol. Soc. Sp. Publ., v. 43, pp. 45-61.

Berman, R. G., 1988, Internally-consistent Thermodynamic data for minerals in the system $\mathrm{Na}_{2} \mathrm{O}-\mathrm{K}_{2} \mathrm{O}-\mathrm{CaO}-\mathrm{MgO}-\mathrm{FeO}-\mathrm{Fe}_{2} \mathrm{O}_{3}$ $\mathrm{Al}_{2} \mathrm{O}_{3}-\mathrm{SiO}_{2}-\mathrm{H}_{2} \mathrm{O}-\mathrm{CO}_{2}$, Jour. Petrol., v. 29, pp. 445-522.

Berman, R. G., 1990, Mixing properties of $\mathrm{Ca}-\mathrm{Mg}-\mathrm{Fe}-\mathrm{Mn}$ garnet. Am. Mineral., v. 75, pp. 328-344.

Bhattacharya, A., Krishna Kumar K. R., Raith M., and Sen S. K., 1991, An improved set off a-x parameters for $\mathrm{Fe}-\mathrm{Mg}-\mathrm{Ca}$ garnets and refinements of the orthopyroxene - garnet theromometer and the orthopyroxene-garnet-plagioclase-quartz barometer. Jour. Petrol., v. 32, pp. 629-656.

Bohlen S. R., Metz G.W., Essene E. J., Anovitz L. M. Westrum E. F., and Wall V. J., 1983a, Thermodynamics and phase equilibria of ferrosilite potential oxygen barometer in mantle rocks, EOS., v. $65, \mathrm{pp} 350$.
Bohlen, S. R., Wall, V. J., and Boettcher A. L., 1983b, Experimental investigation and application of garnet granulite equilibria. Cont. Min. Petrol., v.83, pp. 52-61.

Dahl, P. S., 1980, the thermal compositional dependence of $\mathrm{Fe}+2$ $\mathrm{Mg}+2$ distributions between coexisting garnet and pyroxene: application to geothermometry. Am. Mineral., v. 65, pp 852866.

Eckert, J. O. Jr., Newton R. C., Kleppa O. J., 1991, The $\Delta$ H of reaction and recalibration of garnet - pyroxene - plagioclase - quartz geobarometer in the CMAS systems by solution calorimetry. Am. Mineral., v. 76, pp. 148-160.

Eckert, J. O. Jr., and Newton R. C., 1993, Palaeo-pressure of South India two pyroxene garnet granulites from thermochemically calibrated barometers. Jour. Meta. Geol., v. 11, pp. 845-854.

Harley S. L., 1984, an experimental study of the partitioning of Fe and $\mathrm{Mg}$ between garnet and orthopyroxene. Contrib. and Mineral. and Petrol., v. 86, pp. 359-373.

Holland, T. J. B., and Powell, R., 1985, an internally consistent thermodynamic dataset with uncertainties and correlations: 2 . data and results. Jour. Meta. Geol., v. 3, pp. 343-370.

Holland, T. J. B., and Powell, R., 1985, an enlarged and updated internally consistent thermodynamic data set with uncertainties and correlations: the system $\mathrm{K}_{2} \mathrm{O}-\mathrm{Na}_{2} \mathrm{O}-\mathrm{CaO}-\mathrm{MgO}-\mathrm{FeO}-\mathrm{Fe}_{2} \mathrm{O}_{3}$ $\mathrm{Al}_{2} \mathrm{O}_{3}-\mathrm{TiO}_{2}-\mathrm{SiO}_{2}-\mathrm{C}-\mathrm{H}_{2}-\mathrm{O}_{2}$, Meta. Geol., v. 8, pp. 89-124.

Joshi, M., Thomas, H. and Sharma R. S., 1993, Granulite facies metamorphism in the Achaean gneiss complex from NorthCentral Rajasthan. Proc. Nat. Acad. Sci. India, v. 63 (A), I, pp. 167-187.

Lal, R. K., 1993, Internally consistent recalibrations of mineral equilibria for geothermobarometry involving garnetorthopyroxene-plagioclase-quartz assemblages and their application to the South Indian granulites., Jour. Meta. Geol., v. 11, pp. $855-866$.

Lee, H. Y. and Ganguly, J., 1984, Fe, Mg fractionation between garnet and orthopyroxene: experimental data and application. Geol. Soc. Am. (abs.), No. 52733 (Reprints).

Lee, H. Y. and Ganguly, J., 1988, Equilibrium compositions of coexisting orthopyroxene and garnet: experimental determination in the system $\mathrm{FeO}-\mathrm{MgO}-\mathrm{Al}_{2} \mathrm{O}_{3}-\mathrm{SiO}_{2}$ and application, Jour. Petrol., v. 29, pp. 93-113.

Moecher, D. P., Essene, E. J. and Anovitz, L. M., 1988, Calculation and application of clinopyroxene - garnet - plagioclase - quartz geobarometer., Contrib. Mineral and Petrol., v. 100, pp. 92-106.

Newton, R. C., 1978, Experimental and thermodynamic evidence for the operation of high pressure in Achaean metamorphism. In. Windley, B. F. and Naqvi, S. M. (Eds), Achaean Geochemistry; Elsevier, Amsterdam, pp. 221-240.

Newton, R. C. and Perkins, D., 1982, Thermodynamic calibration of geobarometer based on the assemblages garnet - plagioclase orthopyroxene - (clinopyroxene) - quartz., Am. Mineral., v. 67, pp. 203-222.

Perchuk, L. L., Aranovich L.Ya., Podlesskii K. K., Lavrente'va, I. V., Korsakov, L. P., and Berdnikov, N. V., 1985, Precambrian granulites of the Aldan shield, eastern Siberia, USSR. J. Meta. Geol., v.3, pp. 265-310.

Perchuk, L. L. and Lavrente'va I. V., 1990, Garnet-orthopyroxene and garnet-amphibole geothermobarometry: experimen-tal data and thermodynamics, Int. Geol. Rev., v. 32, pp. 486-507.

Perkins, D. and Chipera, S. J., 1985, Garnet-orthopyroxeneplagioclase-quartz barometery: refinement and application to English River sub province and the Minnesota River valley. Cont. Mineral Petrol., v. 89, pp. 69-80. 
Powell, R. and Holland, T. J. B., 1988, an internally consistent dataset with uncertainties and correlations: 3 Applications to geobarometry. Worked examples and a computer program. Jour. Meta. Geol., v. 6, pp. 173-204.

Raith, M., Raase, P., Ackerman, D. and Lal, R. K., 1983, Regional geothermobarometry in the granulite facies terrain of South India. Transactions of the Royal Society Edinburgh (Earth Sciences), v. 73, pp. 221-244.

Sen, S. K. and Bhattacharya, A., 1984, an orthopyroxene-garnet thermometer and its application to the Madras Charnockite. Contr. Mineral. Petrol., v. 88, pp. 64-71.

Thomas, H., 1995, Pressure Temperature considerations for granulite from Thana Gyangarh, District - Bhilwara, Rajasthan: Implication for crustal evolution. In: R. K. Shrivastava and R. Chandra (Eds.), Magmatism in relation to Divers Tectonic Setting. Oxford and IBH Publishing Com. Pvt. Ltd., pp. 439456.
Thomas, H., 2003, Garnet-orthopyroxene-plagioclase-quartz Equilibria: A comparative study. Gond. Geol. Magz. Advances in Precambrian of Central India., Sp. Vol. no.7, pp. 327-37.

Thomas, H. 2005, Polymetamorphism in the Achaean Gneiss Complex of Shivpura Gyangarh, District Bhilwara, Rajasthan. In: H. Thomas (Ed.), Granulite facies metamorphism and Crustal Evolution., Atlantic publishers and Distributors, New Delhi, pp. 120-146.

Thomas, H., 2006, GARNET.EXE: Calculation of the activity of garnet based on regular and sub-regular solution models using Garent.EXE Software. Jour. Applied Geochem., v. 8 (1), pp. $1-10$.

Thomas, H., 2008, GARNETGS.EXE: A Comparative Study of Activity of Garnet through Sub regular/Regular Ternary Solution Models. Jour. Applied Geochem., v. 8 (1), pp. 31-39.

Wells, P. R. A., 1979, Chemical and thermal evolution of Achaean sialic crust, Southern West Greenland; Jour. Petrol., v. 46, pp. $1-15$. 